\title{
Caracterização de Artefatos Elastoméricos obtidos por Revulcanização de Resíduo Industrial de SBR (Copolímero de Butadieno e Estireno)
}

\author{
Tatiana Weber \\ Instituto Federal de Educação, Ciência e Tecnologia do Rio Grande do Sul, Caxias do Sul, RS \\ Aline Zanchet, Janaina S. Crespo \\ Centro de Ciências Exatas e Tecnologia, UCS \\ Márcia G. Oliveira \\ Divisão de Processamento e Caracterização de Materiais, INT \\ João C. M. Suarez \\ Seção de Engenharia Mecânica e de Materiais, IME \\ Regina C. R. Nunes \\ Instituto de Macromoléculas Professora Eloisa Mano, UFRJ
}

\begin{abstract}
Resumo: A reciclagem de resíduos de borracha é de extrema importância nos dias de hoje, tanto em relação aos impactos ambientais quanto à conservação de energia. Considerando que a maioria dos trabalhos publicados atualmente se refere à reciclagem de pneus, o objetivo deste trabalho foi desenvolver e caracterizar formulações contendo um tipo de resíduo industrial, proveniente da fabricação de perfis automotivos, à base de copolímero de butadieno e estireno (SBR) para possível aplicação na própria empresa. As composições foram preparadas e caracterizadas pela determinação das propriedades térmicas (calorimetria diferencial de varredura), reológicas (analisador de processamento de borracha), reométricas (reômetria de disco oscilatório), mecânicas (resistência à tração e ao rasgamento), químicas (densidade de ligações cruzadas) e morfológicas (microscopia eletrônica de varredura). Os resultados obtidos para as composições foram comparados aos de uma amostra controle, de mesma composição, que foi fornecida pela empresa geradora do resíduo de SBR. Os resultados mecânicos indicaram que o processo de revulcanização proposto foi efetivo. Os valores dos parâmetros de tensão na ruptura e resistência ao rasgamento foram de 70 e $50 \%$, respectivamente, em relação aos valores encontrados para a amostra controle, indicando a possibilidade do uso desse resíduo em artefatos de menor exigência técnica.
\end{abstract}

Palavras-chave: Resíduo industrial de SBR, revulcanização, propriedades mecânicas.

\section{Characterization of Elastomeric Artifacts obtained by Revulcanization of SBR Industrial Waste (Styrene-butadiene Rubber)}

\begin{abstract}
The recycling of waste rubber has very important significances, such as protection of the environment and conservation of energy. Considering that most of published works concerning this subject are about tire recycling, the objective of this work was to develop and characterize formulations containing a type of industrial waste, from the manufacture of automotive profiles, based on styrene-butadiene rubber (SBR) for possible application in the industry. The compositions were formulated and characterized by thermal (differential scanning calorimetry), rheological (rubber processor analyzer), rheometrical (oscillatory disc rheomether), mechanical (tensile strength and tear resistance), chemical (cross-link density) and morphological properties (scanning electron microscopy). The results obtained for these compositions were compared to a control sample purchased for the industry, with the same formulation of the SBR scraps. Obtained results show that the revulcanization process was effective. Values for tensile strength and tear resistance were 70 and $50 \%$, respectively, compared to values found for the control sample, indicating the possibility of using this residue as artifacts of lower technical requirement.

Keywords: SBR industrial waste, revulcanization, mechanical properties.
\end{abstract}

\section{Introdução}

A questão da reutilização de resíduos de borracha, tanto industriais quanto pós-consumo, é de grande importância tanto em relação ao impacto ambiental causado pelos resíduos, quanto à conservação de energia, uma vez que as matérias-primas para a indústria da borracha dependem em grande parte do petróleo ${ }^{[1]}$. Além disso, por possuírem estrutura termorrígida, esses materiais não podem ser fundidos e remoldados como os termoplásticos ${ }^{[2,3]}$.

A maioria dos trabalhos publicados recentemente sobre a reutilização de resíduos de borracha trata de resíduos de pneus ${ }^{[4-12]}$, visto que aproximadamente $70 \%$ de toda borracha consumida é utilizada na produção de pneus. Por este motivo, o estudo da reciclagem de resíduos de borracha na própria indústria, visando a obtenção de um artefato com uma nova aplicação totalmente diferente daquela originalmente proposta, já é uma realidade nas indústrias do setor.

Dentre as borrachas sintéticas mais consumidas pela indústria destaca-se o copolímero de butadieno e estireno (SBR). O SBR é amplamente utilizado na obtenção de pneumáticos, bem como na indústria de artefatos de borracha na produção de perfis expandidos e maciços, de correias para a transmissão de potências,

Autor para correspondência: Janaina S. Crespo, Centro de Ciências Exatas e Tecnologia, Universidade de Caxias do Sul,

Rua Francisco Getúlio Vargas, 1130, CEP 95070-560, Caxias do Sul, RS, Brasil, e-mail: jscrespo@ucs.br 
de correias transportadoras, de mangueiras, de perfilados, de calçados, entre outras ${ }^{[13]}$. A produção desses materiais gera resíduos tanto provenientes do processo produtivo, como resíduos pósconsumo descartados pela sociedade. No Estado do Rio Grande do Sul, a indústria da borracha gera anualmente cerca de 11.800 t de resíduos, o que corresponde a $1 \%$ do total de resíduos industriais gerados no Estado. Desse montante, $87 \%$ são classificados como resíduos industriais Classe II A - não inertes ${ }^{[14]}$. A reciclagem mecânica é uma alternativa de baixo custo para a recuperação de resíduos de borracha industriais, e consiste na moagem dos mesmos para a obtenção de um pó, seguida da reutilização na produção de novos artefatos ${ }^{[15-17]}$.

Dentro desse contexto, esse trabalho teve por objetivo desenvolver e caracterizar formulações vulcanizáveis que tenham aplicação tecnológica, em tapetes e/ou em perfis automotivos obtidos por compressão, com o uso somente de resíduo de SBR gerado no processo industrial de produção de perfis expandidos obtidos por extrusão. O desenvolvimento das formulações foi baseado na revulcanização, e a caracterização foi realizada pela determinação das propriedades térmicas, reológicas, reométricas, mecânicas, químicas e morfológicas.

\section{Experimental}

\section{Obtenção das formulações}

Neste trabalho foram estudadas duas composições elastoméricas visando o aproveitamento, por revulcanização, de resíduos de SBR. As composições foram preparadas a partir de pó de SBR obtido pela moagem de aparas de perfis expandidos produzidos na empresa Ciaflex Indústria de Borrachas Ltda (Caxias do Sul, RS), e que foram coletadas segundo a norma NBR $10.007^{[18]}$. A geração anual média de resíduos de SBR nessa empresa é de 6,6 t/ano. Estes resíduos vêm sendo dispostos na Pró-Ambiente Central de Resíduos Industriais em Gravataí-RS.

O pó de SBR, designado neste trabalho como SBR-r, apresenta as seguintes características: tamanho médio das partículas de 28 a 35 mesh (0,425 a 0,60 mm); composição parcial do resíduo: $30,5 \%$ de SBR, 27,3\% de $\mathrm{CaCO}_{3}, 22,0 \%$ de óleo, 6,5\% de negro de fumo, $13,7 \%$ de massa residual; e teor de gel de $96 \%{ }^{[19]}$.

As formulações das composições que se diferenciam apenas pela adição ou não de óleo aromático foram designadas como A (sem óleo) e B (com 5 partes por cem partes de borracha (phr) de óleo), e possuem os demais componentes em phr: SBR-r (100), enxofre (1), óxido de zinco (5), ácido esteárico (2), inibidor da pré-vulcanização N-ciclohexiltioftalimida (CTP) (1) e N-butilbenzotiazol sulfenamida (TBBS) (1).

As composições foram preparadas à temperatura ambiente em um misturador de rolos $\mathrm{MH}$, modelo $\mathrm{MH}-600$, segundo norma ASTM D 3182-06 $6^{[20]}$, de acordo com o seguinte procedimento: o SBR-r puro (composição A) e a mistura do SBR-r com o óleo aromático (composição B) foram inicialmente processados no misturador durante 5 minutos; após este tempo, foram adicionados os demais componentes e a mistura foi processada no misturador por mais 5 minutos, possibilitando a formação de uma mistura homogênea aderida à superfície do rolo do misturador.

Adicionalmente, foi analisada uma amostra controle com formulação idêntica à das aparas dos perfis de SBR e que foi fornecida pela empresa geradora do resíduo.

Os parâmetros reométricos torque mínimo $\left(\mathrm{M}_{\mathrm{L}}\right)$, torque máximo $\left(\mathrm{M}_{\mathrm{H}}\right)$, tempo de pré-cura $\left(\mathrm{t}_{\mathrm{S1}}\right)$, tempo ótimo de cura $\left(\mathrm{t}_{90}\right)$ e índice de velocidade de cura (CRI - Equação 1) foram obtidos, de acordo com a norma ASTM D 2084-06 ${ }^{[21]}$, em um reômetro de disco oscilatório
(ODR), marca Tech Pro modelo Rheotech, na temperatura de $160{ }^{\circ} \mathrm{C}$ e arco de oscilação de $1^{\circ}$.

$$
C R I=\frac{100}{\left(\mathrm{t}_{90}-\mathrm{t}_{\mathrm{s} 1}\right)}
$$

A revulcanização das composições A e B e a vulcanização da amostra controle foram realizadas através de moldagem por compressão em uma prensa hidráulica (Shultz, modelo PHS 15 T), na temperatura de $160{ }^{\circ} \mathrm{C}$ e pressão de $7,5 \mathrm{MPa}$, no $\mathrm{t}_{90}$ previamente determinado por reometria, conforme norma ASTM 3182-06 ${ }^{[20]}$.

\section{Caracterização das Composições Antes da Revulcanização}

A caracterização das composições antes da revulcanização foi realizada por meio de calorimetria diferencial de varredura (DSC) e pela determinação da viscosidade dinâmica complexa $\left(\eta^{*}\right)$ em função da taxa de cisalhamento.

A análise de DSC foi realizada em um equipamento Shimadzu, modelo DSC-50. As amostras ( 10 mg) foram aquecidas com uma taxa de aquecimento de $30{ }^{\circ} \mathrm{C} / \mathrm{min}^{-1}$, da temperatura ambiente até $300{ }^{\circ} \mathrm{C}$ sob um fluxo de nitrogênio de $50 \mathrm{~mL} / \mathrm{min}^{-1}$. As temperaturas de transição foram determinadas, visando uma avaliação das características de cura.

A viscosidade dinâmica complexa $\left(\eta^{*}\right)$ em função da taxa de cisalhamento foi determinada a partir do ensaio de variação de frequência em um analisador de processamento de borracha marca Alpha Technology, modelo RPA 2000, de acordo com a norma ASTM D 6204-07 $7^{[22]}$. O ensaio foi executado variando-se a frequência de 0,0033 a $16,667 \mathrm{~Hz}$, na temperatura de $100{ }^{\circ} \mathrm{C}$ e com $28 \%\left(2^{\circ}\right)$ de deformação. A faixa escolhida para a taxa de cisalhamento é equivalente a do processo de moldagem por compressão ${ }^{[23]}$.

\section{Caracterização das Composições após a Revulcanização}

A caracterização das composições estudadas após revulcanização foi realizada através da determinação da densidade de ligações cruzadas, por meio de ensaios mecânicos (de tração e de rasgamento) e por análise fratográfica.

A densidade de ligações cruzadas [X] foi calculada utilizando-se a equação de Flory-Rehner ${ }^{[24-26]}$ (Equação 2) a partir do inchamento no equilíbrio.

$$
[X]=\frac{-\left[\ln \left(1-v_{r}\right)+v_{r}+\chi \cdot v_{r}^{2}\right]}{V_{o}\left(v_{r}^{1 / 3}-v_{r} / 2\right)}
$$

Onde:

- $v_{\mathrm{r}}$ é a fração volumétrica de borracha inchada;

- $\chi$ é o parâmetro de interação borracha-solvente (SBR-tolueno $\left.0,31^{[27]}\right) ; \mathrm{e}$

- $\mathrm{V}$ é o volume molar do solvente, $\mathrm{em} \mathrm{cm}^{3} \cdot \mathrm{mol}^{-1}$ (tolueno $\left.105,91 \mathrm{~cm}^{3} \cdot \mathrm{mol}^{-1[27]}\right)$.

Foram utilizadas amostras de dimensões em torno de $20 \times 20 \times 2 \mathrm{~mm}$, imersas em tolueno, no escuro e à temperatura ambiente. As amostras foram pesadas diariamente até que não houvesse mais variação de massa, o que levou cinco dias. Em seguida, as amostras foram secas em estufa a vácuo a $60{ }^{\circ} \mathrm{C}$ por 4 horas. A determinação foi realizada em duplicata.

Os ensaios mecânicos, de tração e rasgamento, foram realizados à $23 \pm 2{ }^{\circ} \mathrm{C}$ e com umidade controlada $(50 \pm 5 \%)$, em uma máquina universal de ensaios marca EMIC, modelo DL3000, com célula de carga de $20 \mathrm{kN}$, de acordo com as normas ASTM D 412-06 $6^{[28]}$ e ASTM D 624-00 ${ }^{[29]}$, respectivamente. Os corpos de prova para os ensaios foram fabricados por corte mecânico a frio, a partir das placas revulcanizadas tendo sido testados na velocidade de 
$500 \mathrm{~mm} / \mathrm{min}^{-1}$. Nos ensaios mecânicos de tração e rasgamento foram ensaiados cinco corpos de prova por composição, calculandose um valor médio para cada propriedade.

A análise fratográfica foi realizada em um microscópio eletrônico de varredura marca JEOL, modelo JSM 5800LV, pela observação direta da topografia das superfícies de fratura de amostras fraturadas, após imersão em nitrogênio líquido, e de corpos de prova ensaiados em tração. Foram determinados, em cada situação, os mecanismos de fratura, procurando-se correlacionar os modos de fratura predominantes com as modificações resultantes do processo de revulcanização e com os resultados numéricos do ensaio de tração. As amostras, antes do exame microscópico, foram recobertas com ouro em uma câmara a vácuo.

\section{Resultados e Discussão}

\section{Determinação das características reométricas}

Os parâmetros de vulcanização para a amostra controle e para as composições A e B encontram-se na Tabela 1.

As composições A e B apresentam, em relação à amostra controle, maiores valores de torque máximo $\left(\mathrm{M}_{\mathrm{H}}\right)$, de torque mínimo $\left(\mathrm{M}_{\mathrm{L}}\right)$ e de tempo de vulcanização $\left(\mathrm{t}_{90}\right)$, menor tempo de segurança de processo $\left(\mathrm{t}_{\mathrm{s} 1}\right)$, assim como menor taxa de vulcanização (CRI). Sabe-se que o $\mathrm{M}_{\mathrm{H}}$ está relacionado à rigidez molecular sendo considerado como uma medida da densidade das ligações cruzadas e da susceptibilidade da ocorrência de reticulação, e que o $M_{L}$ pode ser relacionado diretamente com a viscosidade de compostos não vulcanizados ${ }^{[30]}$. Os resultados obtidos indicam que as composições A e B apresentam, em relação à amostra controle, um retardo no processo de vulcanização e um processamento mais difícil devido, provavelmente, a maior quantidade de ligações cruzadas e às viscosidades mais elevadas. Observa-se ainda que a composição $\mathrm{A}$ apresenta, em relação à composição $\mathrm{B}$, parâmetros reométricos com valores mais elevados, com exceção do $t_{90}$ que é menor. Estes resultados indicam que a adição de óleo aromático facilita o processamento da composição B, mas que o término do processo de vulcanização deve sofrer um retardo, conforme indicado pelo $t_{90}$ da composição.

\section{Caracterização antes da revulcanização}

\section{Calorimetria diferencial de varredura (DSC)}

A temperatura e as entalpias das transições endotérmicas e exotérmicas do SBR-r, da amostra controle e das composições A e B, determinados por DSC, estão sumarizados na Tabela 2. Como pode ser observado, o SBR-r apresenta apenas um pico endotérmico, enquanto que as composições A e B apresentam, além do pico endotérmico, um pico exotérmico. Além disso, a amostra controle apresenta apenas o pico exotérmico.

Tabela 1. Parâmetros de vulcanização da amostra controle e das composições A e B.

\begin{tabular}{cccc}
\hline Propriedade & \multicolumn{3}{c}{ Amostra } \\
\cline { 2 - 4 } & Controle & A & B \\
\hline $\mathrm{M}_{\mathrm{H}}(\mathrm{dN} \mathrm{m})$ & 48,1 & 108,6 & 72,2 \\
$\mathrm{M}_{\mathrm{L}}(\mathrm{dN} \mathrm{m})$ & 13,5 & 53,3 & 38,2 \\
$\Delta \mathrm{M}(\mathrm{dN} \mathrm{m})$ & 34,6 & 55,3 & 34,0 \\
$\mathrm{t}_{90}(\min )$ & 10,8 & 17,8 & 27,4 \\
$\mathrm{t}_{\mathrm{S} 1}(\min )$ & 3,5 & 2,1 & 1,4 \\
$\mathrm{CRI}\left(\mathrm{min}^{-1}\right)$ & 13,6 & 6,3 & 3,8 \\
\hline
\end{tabular}

A ocorrência do pico endotérmico, em torno de $100{ }^{\circ} \mathrm{C}$, está relacionada à fusão do enxofre residual presente no SBR-r ${ }^{[31]}$. $\mathrm{O}$ aumento observado nas entalpias de fusão das composições A e B, quando comparadas com a do SBR-r, pode ser atribuído à maior quantidade de enxofre existente nas mesmas. Verificase, adicionalmente, que o sistema de cura das composições A e $\mathrm{B}$, constituído por um acelerador (TBBS), dois ativadores (ácido esteárico e $\mathrm{ZnO}$ ) e um inibidor de pré-vulcanização (CTP), influencia a temperatura de fusão do enxofre; o aumento da razão acelerador/enxofre reduz a temperatura de fusão do enxofre, que passa de $105,5{ }^{\circ} \mathrm{C}$ no SBR-r para $94{ }^{\circ} \mathrm{C}$ em A, e $92,8^{\circ} \mathrm{C}$ em B.

A presença do pico exotérmico nas composições A e B indica que ocorre, com o aumento da temperatura, uma vulcanização adicional, que é confirmada uma vez que em uma segunda corrida por DSC não ocorre o aparecimento do pico exotérmico ${ }^{[5,32]}$. A diferença observada nas temperaturas de vulcanização (cerca de $139^{\circ} \mathrm{C}$ para a amostra controle e, aproximadamente, $195^{\circ} \mathrm{C}$ para as composições A e B), está provavelmente relacionada com a adição do CTP que é um inibidor de pré-vulcanização à essas composições, o que retarda o processo de vulcanização das mesmas.

Verifica-se ainda que o valor de entalpia da amostra controle é maior do que as das composições A e B. Esta diferença pode ser explicada considerando que a amostra controle apresenta uma maior quantidade de ligações duplas na cadeia polimérica que são passíveis de vulcanização, enquanto que as composições A e B apresentam, na sua composição, o SBR-r que se encontra praticamente vulcanizado, com um teor de gel de $96 \%{ }^{[19]}$. A composição B, quando comparada com à A, apresenta uma maior entalpia devido ao óleo existente na sua composição. O óleo, ao aumentar o afastamento entre as cadeias, exige que o rompimento da barreira de flexibilidade, necessária para a reação de vulcanização, ocorra com maior energia. Esta observação está de acordo com o maior $\mathrm{t}_{90}$ determinado para a composição B.

Tabela 2. Resultados de DSC do SBR-r, da amostra controle e das composições A e B.

\begin{tabular}{cccccc}
\hline \multirow{2}{*}{ Amostra } & \multicolumn{2}{c}{ Pico endotérmico } & & \multicolumn{2}{c}{ Pico exotérmico } \\
\cline { 2 - 3 } \cline { 5 - 6 } & $\begin{array}{c}\text { Temperatura } \\
\left({ }^{\circ} \mathbf{C}\right)\end{array}$ & $\begin{array}{c}\text { Energia } \\
\left(\mathbf{J . g}^{-1}\right)\end{array}$ & & $\begin{array}{c}\text { Temperatura } \\
\left({ }^{\circ} \mathbf{C}\right)\end{array}$ & $\begin{array}{c}\text { Energia } \\
\left(\mathbf{J . g}^{-1}\right)\end{array}$ \\
\hline SBR-r & 105,5 & 3,4 & & - & - \\
Controle & - & - & & 138,7 & 9,3 \\
A & 94,0 & 8,4 & & 194,7 & 3,7 \\
B & 92,8 & 7,8 & & 191,1 & 5,1 \\
\hline
\end{tabular}

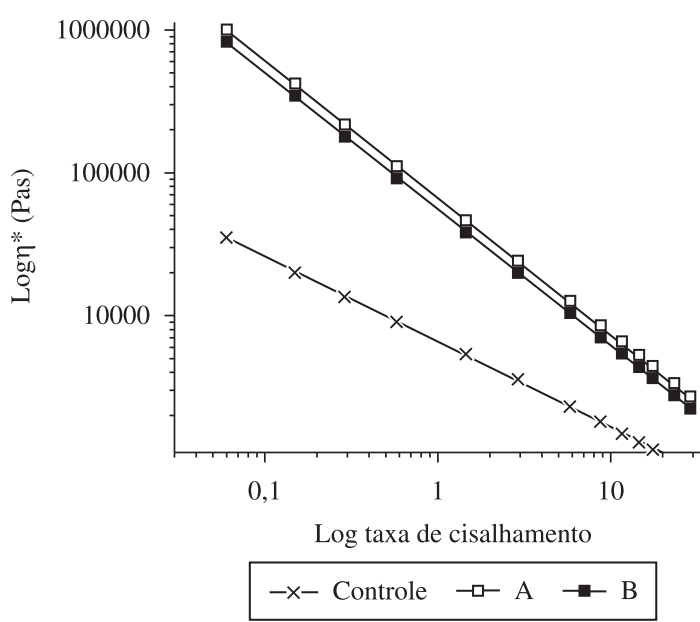

Figura 1. Viscosidade dinâmica complexa $v s$. taxa de cisalhamento da amostra controle e das composições A e B. 
Tabela 3. Resultados da densidade de ligações cruzadas e dos ensaios mecânicos da amostra controle e das composições A e B.

\begin{tabular}{cccc}
\hline Propriedade & \multicolumn{3}{c}{ Amostra } \\
\cline { 2 - 4 } & Controle & A & B \\
\hline Densidade de ligações cruzadas (mol.cm ${ }^{-3}$ ) & $2,99 \times 10^{-4} \pm 0,338 \times 10^{-4}$ & $4,28 \times 10^{-4} \pm 0,132 \times 10^{-4}$ & $5,01 \times 10^{-4} \pm 0,004 \times 10^{-4}$ \\
Tensão na ruptura (MPa) & $3,2 \pm 0,6$ & $2,1 \pm 0,2$ & $2,2 \pm 0,2$ \\
Alongamento na Ruptura (\%) & $443,0 \pm 32,2$ & $73,3 \pm 7,1$ & $98,0 \pm 11,8$ \\
Módulo a 50\% (MPa) & $0,4 \pm 0,1$ & $1,7 \pm 0,1$ & $1,6 \pm 0,1$ \\
Resistência ao rasgamento $\left(\mathrm{kN} . \mathrm{m}^{-1}\right)$ & $16,2 \pm 3,4$ & $7,5 \pm 1,3$ & $8,1 \pm 1,3$ \\
\hline
\end{tabular}

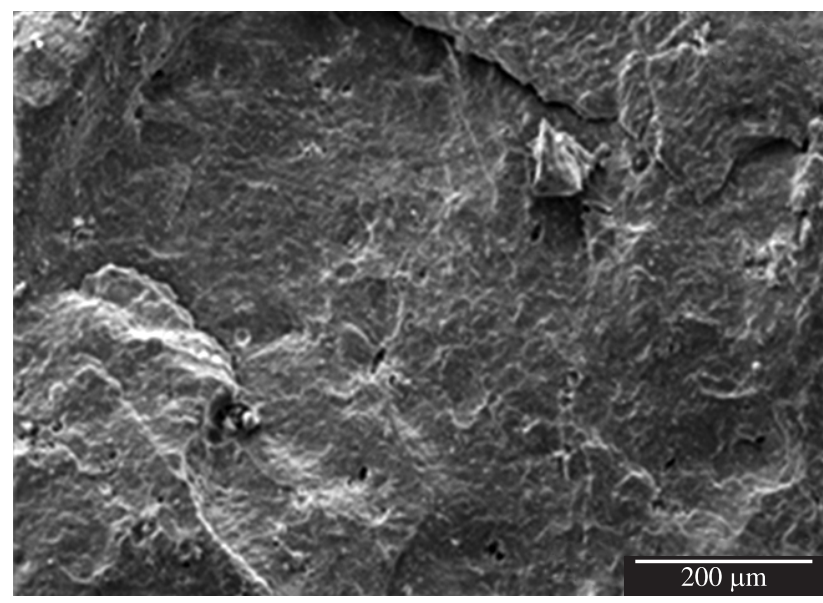

(a)

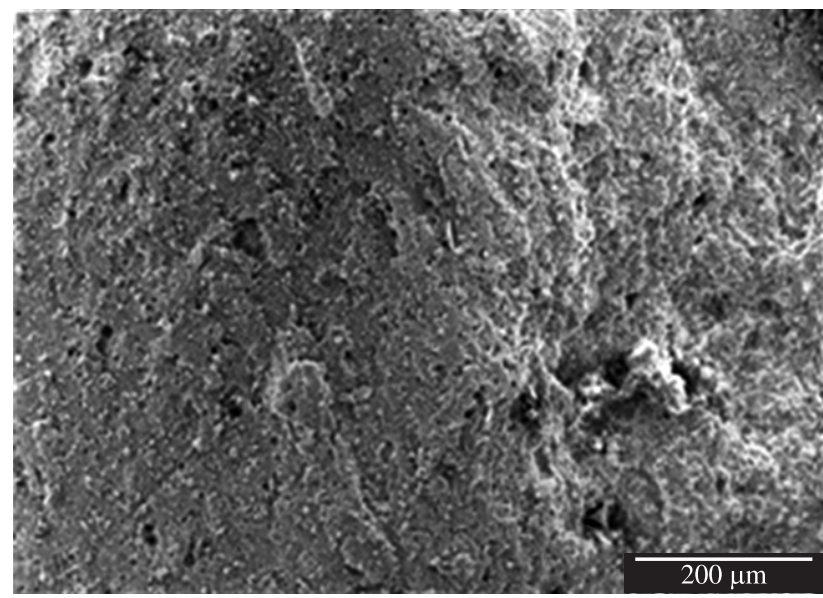

(c)

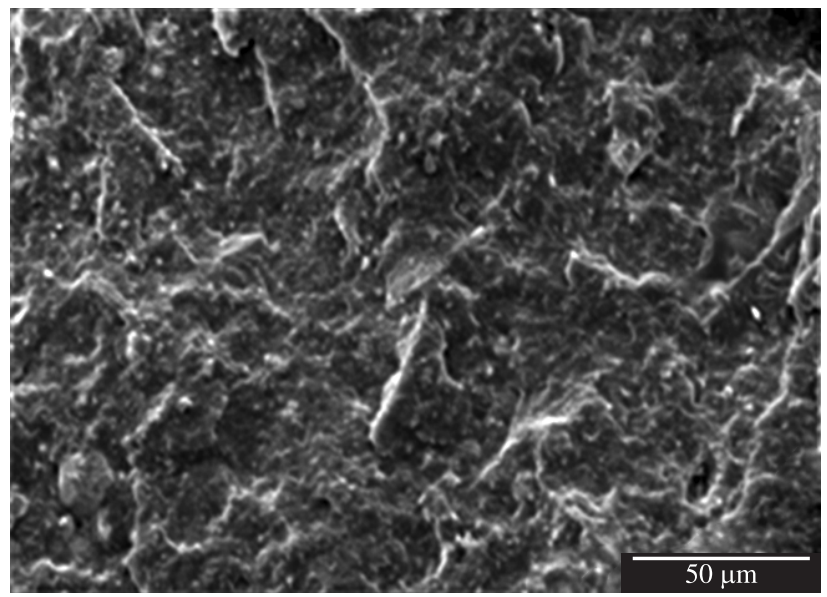

(b)

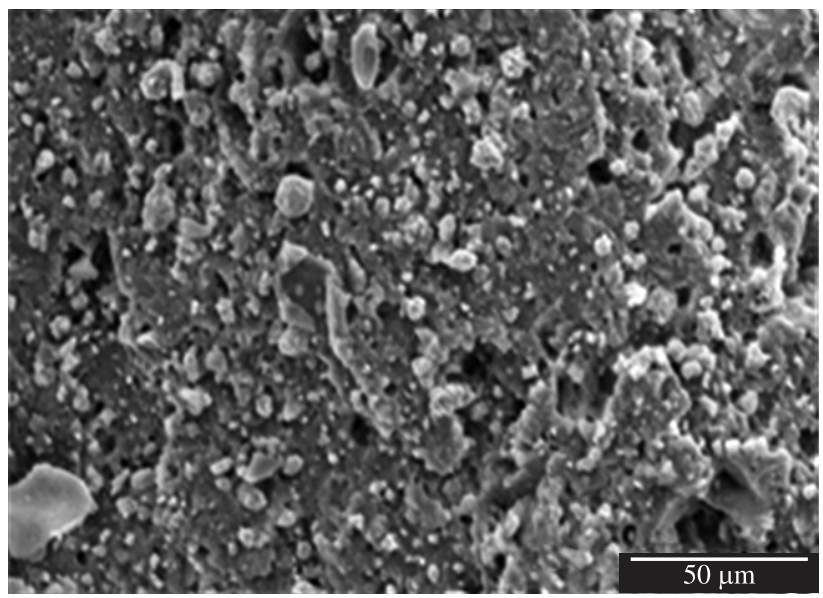

(d)

Figura 2. Microfotografias por MEV das superfícies de fratura de corpos de prova da amostra controle: a) criofraturados; (b) detalhe de (a); c) ensaiados em tração; e d) detalhe de (c).

Determinação da viscosidade dinâmica complexa $\left(\eta^{\star}\right)$ em função da taxa de cisalhamento

A Figura 1 apresenta a variação da viscosidade dinâmica em função da taxa de cisalhamento, determinada no analisador de processamento de borracha, para a amostra controle e as composições A e B. Observa-se que a viscosidade diminui com o aumento de taxa de cisalhamento, o que indica que as três composições apresentam um comportamento pseudoplástico ${ }^{[23]}$. As composições A e B, quando comparadas com a amostra controle, apresentam maiores viscosidades que variam de uma maneira mais acentuada. A composição B é um pouco menos viscosa do que a A, devido ao óleo aromático adicionado a esta composição. O comportamento apresentado pelas composições A e B indica que as mesmas deverão ter, em relação à amostra controle, um processamento mais difícil e menos estável. Estes resultados são corroborados pelos valores obtidos para os parâmetros reométricos da amostra controle e das composições A e B.

\section{Caracterização após a revulcanização}

Densidade de ligações cruzadas e ensaios mecânicos

Os resultados de densidade de ligações cruzadas e dos ensaios mecânicos da amostra controle e das composições A e B estão apresentados na Tabela 3. Verifica-se que as composições A e B apresentam, em relação à amostra controle, maiores valores de densidade de ligações cruzadas. Os altos valores encontrados para as composições $\mathrm{A}$ e $\mathrm{B}$ podem ser relacionados com as maiores quantidades de enxofre e de acelerador existentes nas mesmas e que 
levam à formação de uma maior quantidade de ligações cruzadas, e também ao fato do SBR-r ser um resíduo vulcanizado.

No ensaio de resistência à tração observa-se que as propriedades das composições A e B, à exceção do módulo a 50\%, são inferiores às da amostra controle. Esses valores, especialmente os referentes ao alongamento na ruptura, caracterizam que as composições A e B apresentam em relação à amostra controle, menor resistência e uma maior fragilidade (menor elasticidade). Esses resultados indicam que, provavelmente, ocorreu uma degradação parcial do elastômero durante o processo de moagem do resíduo. Observa-se, todavia, que as composições A e B são muito mais rígidas, pois quando comparadas com a amostra controle, apresentam um módulo a 50\% cerca de quatro vezes maior. A rigidez das composições A e B é devida à maior densidade de ligações cruzadas, o que é corroborado pelos valores de $\mathrm{M}_{\mathrm{H}}$, obtidos na análise reométrica.

A comparação dos valores de tensão na ruptura e de resistência ao rasgamento das composições desenvolvidas, com os da amostra controle, indica que foi alcançada uma recuperação da ordem de 70 e 50\%, respectivamente. Os resultados obtidos são promissores, uma vez que não houve adição de borracha virgem e pelo fato de existirem registros na literatura ${ }^{[33,34]}$ que consideram aceitáveis composições regeneradas com $30 \%$ das propriedades originais.

\section{Análise fratográfica}

A Figura 2 apresenta microfotografias por MEV das superfícies de fratura de corpos de prova da amostra controle, criofraturados e ensaiados em tração. Verifica-se que a amostra controle criofraturada (Figuras 2a,b) tem uma superfície de fratura homogênea, com baixa rugosidade e linhas de rasgamento. A amostra controle ensaiada em tração mostra, em pequeno aumento (Figura 2c), apresenta uma superfície de fratura relativamente plana, bastante semelhante à normalmente observada nos materiais elastoméricos. Em maior aumento (Figura 2d), pode-se observar a presença de numerosos aglomerados, aproximadamente esféricos, distribuídos na superfície de fratura, mostrando que a fratura em tração da amostra controle ocorreu com grande deformação plástica.

Na Figura 3 estão as microfotografias por MEV das superfícies de fratura de corpos de prova criofraturados das composições A e B. As amostras, independentemente da composição, mostram sob

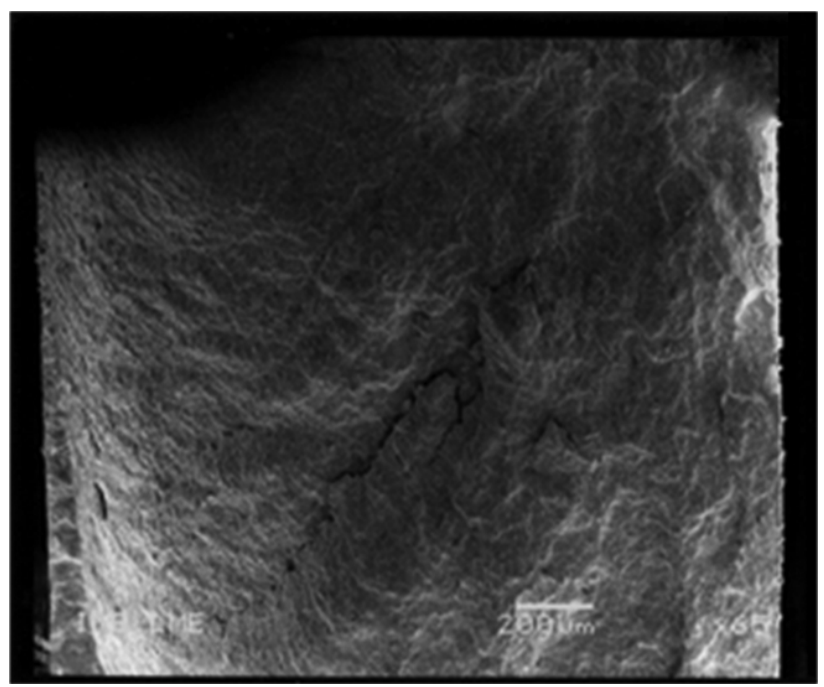

(a)

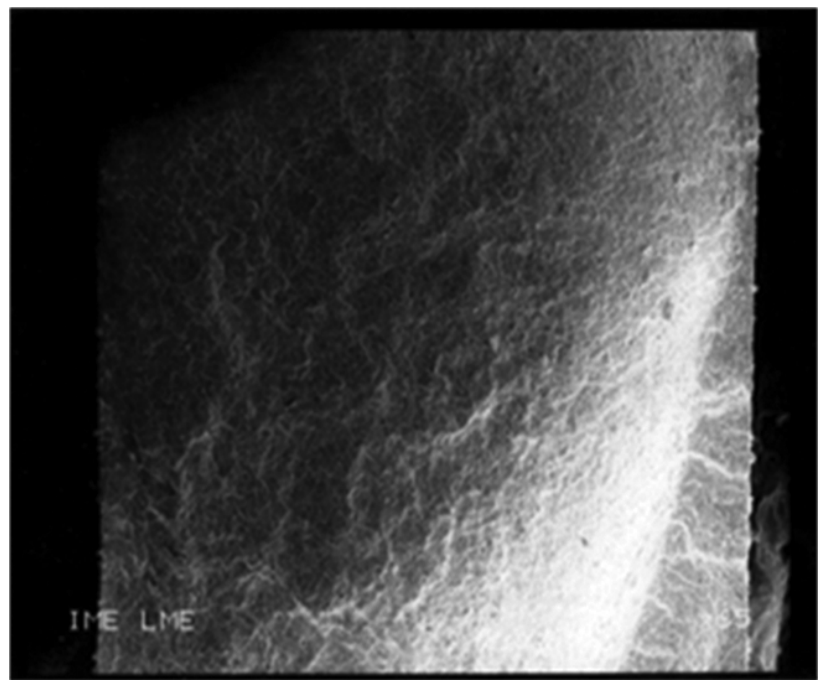

(c)

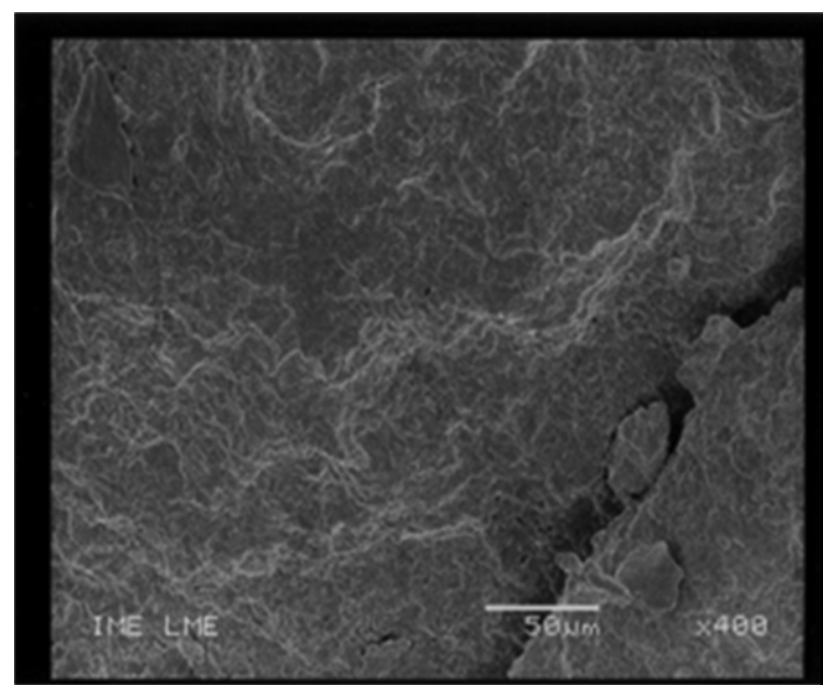

(b)

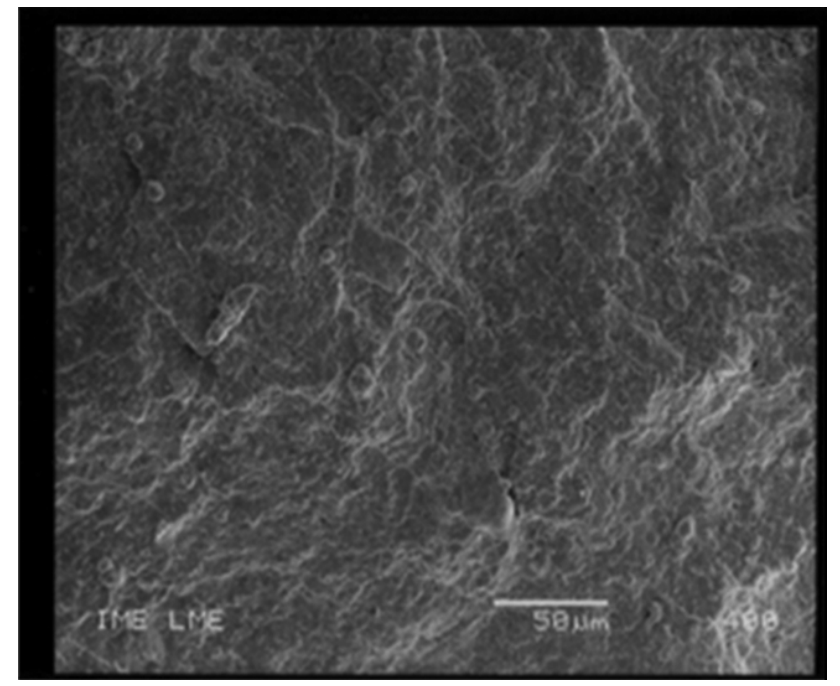

(d)

Figura 3. Microfotografias por MEV das superfícies de fratura de corpos de prova criofraturados das composições recicladas: a) composição A; b) detalhe de (a); c) composição B; e d) detalhe de (c). 


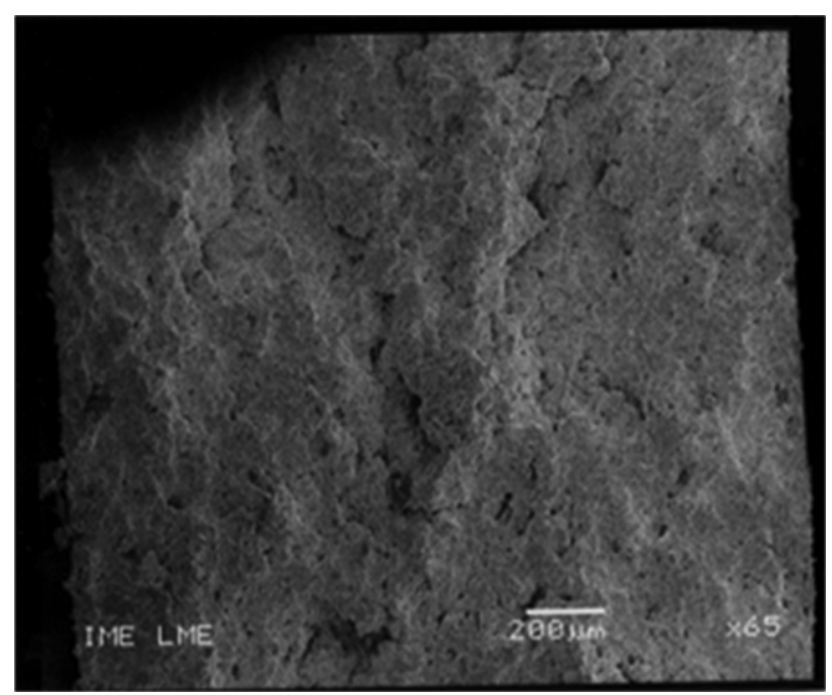

(a)

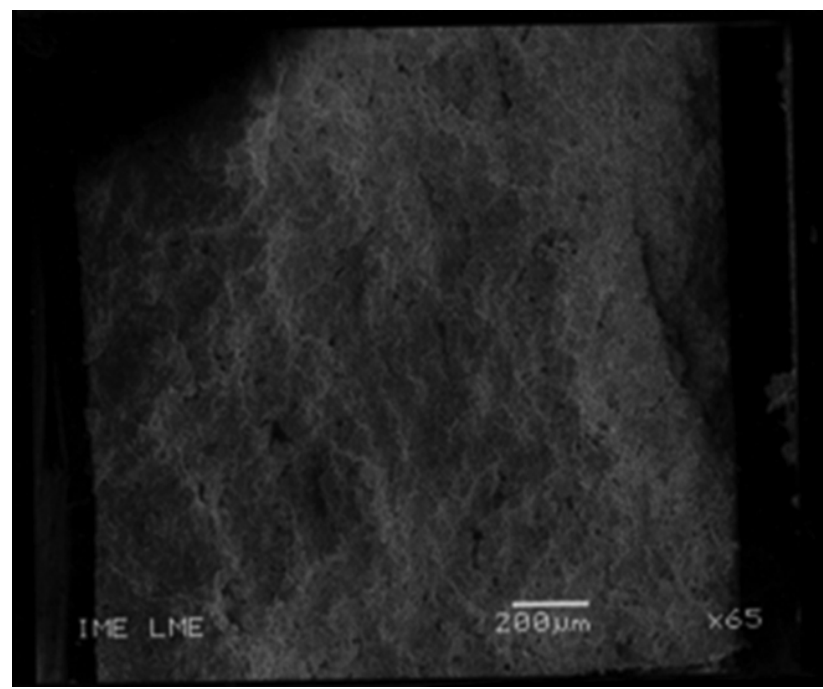

(c)

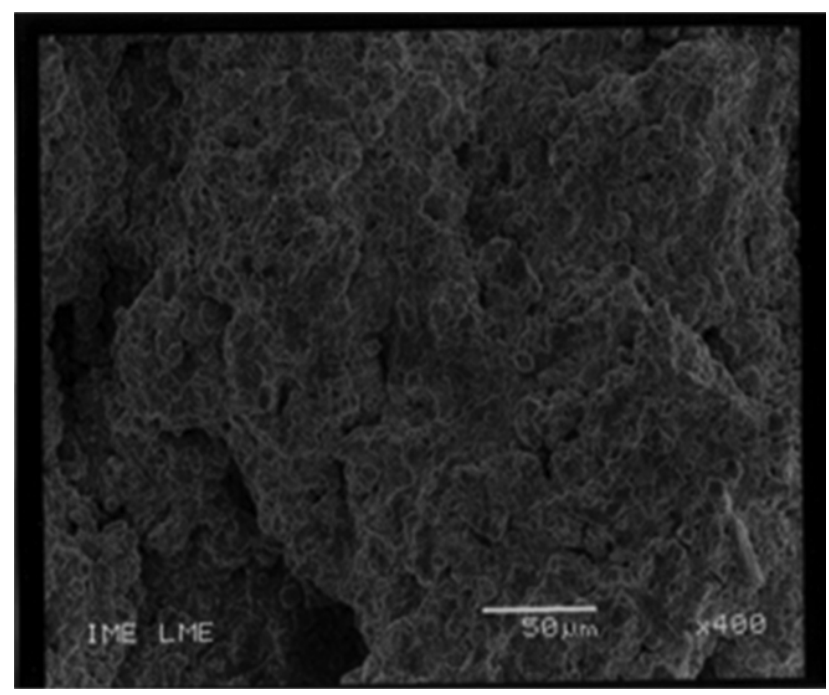

(b)

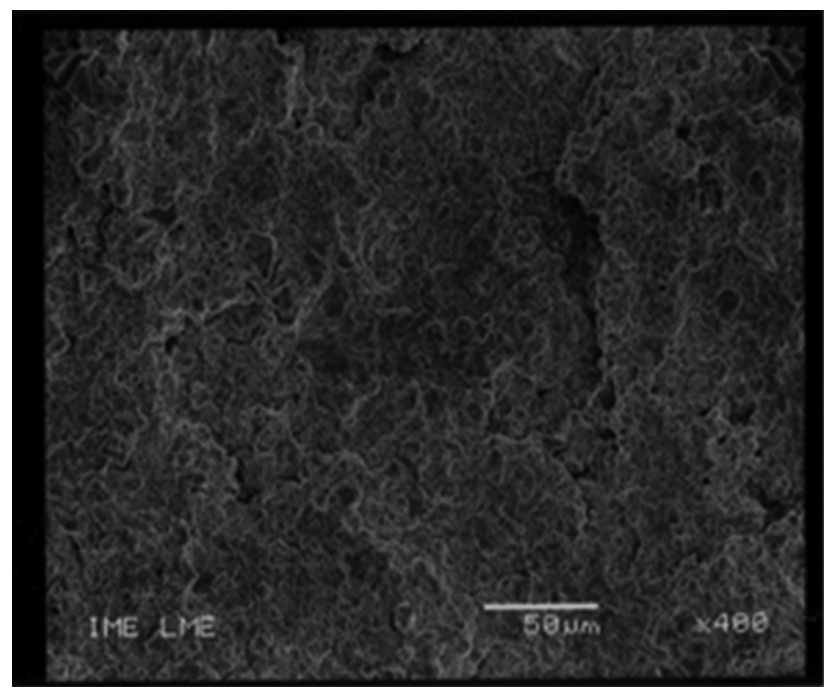

(d)

Figura 4. Microfotografias por MEV das superfícies de fratura de corpos de prova ensaiados em tração das composições recicladas: a) composição A; b) detalhe de (a); c) composição B; e d) detalhe de (c).

menores aumentos (Figuras 3a,c), aspectos topográficos semelhantes, superfícies de fratura pouco rugosas com linhas de rasgamento. No exame com maiores aumentos (Figuras 3b,d), verifica-se a existência de trincas e inclusões, especialmente na composição A.

De maneira geral, pode-se considerar que as duas composições recicladas apresentam, entre si e quando comparadas com a amostra controle, o mesmo modo dominante de fratura. Embora o modo de fratura das três composições seja idêntico, as composições recicladas em relação à amostra controle, são mais frágeis e deverão ter uma menor plasticidade.

As microfotografias por MEV das superfícies de fratura de corpos de prova ensaiados em tração das composições A e B estão na Figura 4. O exame com pequeno aumento das composições A e B (Figuras 4a,c, respectivamente) mostra que as mesmas apresentam superfícies de fratura com aspectos microscópicos similares, superfície rugosa, sulcos, vazios e trincas. Sob maiores aumentos, é possível observar que a composição A (Figura 4b), apresenta, em relação à composição B (Figura 4d), uma superfície de fratura mais grosseira, com um relevo mais acentuado, facetas em forma de escamas e maior quantidade de trincas. Estes aspectos sugerem que as composições recicladas fraturaram com baixa plasticidade por um processo descontínuo de fratura. A análise microscópica das superfícies de fratura da amostra controle e das composições A e B está de acordo com os resultados numéricos do ensaio de tração e corrobora o comportamento das composições de SBR reciclado.

\section{Conclusões}

Neste trabalho foi estudado o reaproveitamento de resíduo de SBR gerado no processo industrial de perfis automotivos. A revulcanização desse resíduo, com base no novo sistema de cura proposto, torna possível sua reutilização em um artefato de menor exigência técnica, como tapetes e/ou perfis automotivos que podem ser obtidos pelo processo de compressão. O controle na formação de novas ligações cruzadas foi fundamental para o desempenho mecânico e reológico adequados para a obtenção de peças moldadas por compressão. Isto pode ser justificado pelos resultados dos ensaios mecânicos das composições A e B, que ficaram próximos aos da amostra controle. 


\section{Agradecimentos}

Os autores agradecem à Ciaflex Indústria de Borrachas Ltda pelo fornecimento dos resíduos de SBR e do material de consumo, e à FAPERGS (PqG 06/2010 - 1006663) e ao CNPq (302924/2010-8) pelo apoio financeiro.

\section{Referências Bibliográficas}

1. Fang, Y.; Zhan, M. \& Wang, Y. - Mater. Design, 22, p.123 (2001). http://dx.doi.org/10.1016/S0261-3069(00)00052-2

2. Adhikari, B.; De, D. \& Maiti, S. - Prog. Polym. Sci., 25, p.909 (2000). http://dx.doi.org/10.1016/S0079-6700(00)00020-4

3. Myhre, M. \& Mackillop, D. A. - Rubber Chem. Technol., 75, p.429 (2002). http://dx.doi.org/10.5254/1.3547678

4. Garcia, I. T. S.; Nunes, M. R.; Carreño, N. L. V.; Wallaw, W. M.; Fajardo, H. V. \& Probst, L. F. D. - Polímeros, 17, 4, p.329 (2007).

5. Gujel, A. A.; Brandalise, R. N.; Giovanela, M.; Crespo, J. S. \& Nunes, R. C. R. - Polímeros, 18, p.320 (2008).

6. Lin, C.; Huang, C.-L. \& Shern, C.-C. - Resour. Conserv. Recy., 52, p.1162, (2008). http://dx.doi.org/10.1016/j.resconrec.2008.06.003

7. Lagarinhos, C. A. F. \& Tenório, J. A. S. - Polímeros, 18, 2, p.106 (2008).

8. Cossa, M. M.; Sirqueira, A. S. \& Soares, B. G. - Polímeros, 19, 3, p.190 (2009).

9. Yilmaz, A. \& Degirmenci, N. - Waste Manage., 29, p.1541 (2009). PMid:19110410. http://dx.doi.org/10.1016/j.wasman.2008.11.002

10. Wu, B. \& Zhou, M. H. - Waste Manage., 29, p.355 (2009). http:// dx.doi.org/10.1016/j.wasman.2008.03.002

11. Zhang, S. L.; Zhen, Z. X.; Xin, Z. X.; Pal, K. \& Kim, J. K. Mater. Design, 31, p.1900 (2010). http://dx.doi.org/10.1016/j. matdes.2009.10.057

12. Zhang, S. L.; Zhen, Z. X.; Pal, K.; Xin, Z. X.; Suh, J. \& Kim, J. K. - Mater. Design, 31, p.3624 (2010). http://dx.doi.org/10.1016/j. matdes.2010.02.039

13. Lovison, V. M. H.; Rocha, E. C. \& Pierozan, N. J. - "Tecnologia de Transformação dos Elastômeros", SENAI, Centro Tecnológico de Polímeros, São Leopoldo (2003).

14. Fundação Estadual de Proteção Ambiental - FEPAM. - "Relatório sobre a geração de resíduos sólidos industriais no Estado do Rio Grande do Sul", FEPAM 2003. Disponível em: <http://www.fepam. rs.gov.br/biblioteca/rsi.asp>. Acesso em: 21 set. 2010.

15. Weber, T.; Oliveira, M. G.; Zeni, M.; Crespo, J. S. \& Nunes, R. C. R. - Polym. Bull., 61, p.217 (2008). http://dx.doi.org/10.1007/s00289008-0937-3

16. Carli, L. N.; Boniatti, R.; Teixeira, C. E.; Nunes, C. R. C. \& Crespo, J. S. - Mat. Sci. Eng. C - Bio S, 29, p.383 (2009).

17. Zanchet, A.; Dal'Acqua, N.; Weber, T.; Crespo, J. S.; Brandalise, R. N. \& Nunes, R. C. R. - Polímeros, 17, 1, p.23 (2007).
18. Associação Brasileira de Normas Técnicas - ABNT. - "NBR 10.007: Amostragem de resíduos sólidos", ABNT 2004.

19. Weber, T.; Zanchet, A.; Brandalise, R. N.; Crespo, J. S. \& Nunes, R. C. R. - J. Elastom. Plast., 40, p.147 (2008). http://dx.doi. org/10.1177/0095244307082487

20. American Society for Testing and Material Standards - ASTM. "ASTM D 3182: Standard practice for rubber - materials, equipment, and procedures for mixing standard compounds and preparing standard vulcanized sheets", ASTM 2006.

21. American Society for Testing and Material Standards - ASTM. - "ASTM $D$ 2084: Standard test method for rubber property - vulcanization using oscillating disk meter", ASTM 2006.

22. American Society for Testing and Material Standards - ASTM. - "ASTM $D$ 6204: Standard test method for rubber-measurement of unvulcanized rheological properties using rotorless shear rheometers", ASTM 2007.

23. Bretas, R. E. S. \& D'Ávila M. A. - "Reologia de Polímeros Fundidos", EdUFSCAR, São Carlos (2000).

24. Saville, B. \& Watson, A. A. - Rubber Chem. Technol., 40, p.100 (1967). http://dx.doi.org/10.5254/1.3539039

25. Yu, V.; Kim, S. H. \& Isayev, A. I. - Rubber Chem. Technol. 70, p.641 (1997).

26. Flory, P. J. - "Principles of Polymer Chemistry", Cornel University, New York (1953).

27. Brandrup, J.; Immergut, E. H.; Grulke, E. A. - "Polymer handbook", Wiley-Interscience Publication, New York (1999).

28. American Society for Testing and Material Standards - ASTM. - "ASTM $D$ 412: Standard test methods for vulcanized rubber and thermoplastic elastomers - tension", ASTM 2006.

29. American Society for Testing and Material Standards - ASTM. “ASTM D 624: Standard test method for tear strength of conventional vulcanized rubber and thermoplastic elastomers, EUA, 2000.

30. Castro, D. F.; Suarez, J. C. M.; Nunes, R. C. R. \& Visconte, L. L. Y. - J. Appl. Polym. Sci., 90, p. 2156 (2003). http://dx.doi.org/10.1002/ app. 12856

31. Weast, R. C.- "CRC Handbook of Chemistry and Physics", CRC Press, New York (1980).

32. Peres, A. C. C.; Lopes, L. M. A.; Visconte, L. L. Y. \& Nunes, R. C. R. - Polímeros, 16, 1, p.61 (2006).

33. Ishiaku, U. S.; Chong, C. S. \& Ismail, H. - Polym. Test., 18, p.621 (1999). http://dx.doi.org/10.1016/S0142-9418(98)00060-9

34. Zanchet, A.; Carli, L. N.; Giovanela, M.; Crespo, J. S.; Scuracchio, C. H. \& Nunes, R. C. R. - J. Elastom. Plast., 41, p.497 (2009). http:// dx.doi.org/10.1177/0095244309345411

Enviado: 11/11/10

Reenviado: 23/03/11

Aceito: $27 / 03 / 11$

DOI: $10.1590 / \$ 0104-14282011005000066$ 